\title{
POTENCIAL DE CRESCIMENTO DE MACROALGAS CULTIVÁVEIS PRESENTES NA ENSEADA DE ARMAÇÃO DO ITAPOCOROY (PENHA, SC): AVALIAÇÃO PRELIMINAR
}

\author{
CUNHA,S.R. ${ }^{1,2}$; PAZETO,F.D.'; CRESTANI,D.E.V.'; LIMA,G.B. ${ }^{1}$; NASCIMENTO,J. ${ }^{1,3}$; \\ SANT'ANNA,F.'; MANZONI, G.C. '; MARENZI, A. W. C. ${ }^{1}$ \& MAFRA Jr.,L.L.'1 \\ ${ }^{1}$ Centro de Ciências Tecnológicas, da Terra e do Mar - Universidade do Vale do Itajaí \\ (CTTMar/UNIVALI) Rua Uruguai, 458 - Cx.Postal 360, Cep: 88.302-202 - Itajaí, SC. \\ e-mail:srcunha@univali.rct-sc.br \\ 2 Universidade do Rio Grande, Lab. Comunidades Vegetais Costeiras (FURG). \\ ${ }^{3}$ Bolsista ProBIC-UNIVALI
}

\begin{abstract}
RESUMO
Este trabalho teve como objetivo testar a metodologia de crescimento e avaliar o potencial de cultivo das algas vermelhas produtoras de ágar, Gracilaria cervicornis (cf.) e Gracilaria caudata, e da produtora de carragenana, Hypnea musciformis. O crescimento in vitro foi testado para as três espécies e o crescimento in situ foi testado para Gracilaria cervicornis (cf.). Os resultados preliminares indicam taxas de crescimento elevadas quando comparadas com a literatura, tanto para os experimentos de laboratório, quanto de campo. As taxas de crescimento de Gracilaria cervicornis (cf.) variaram entre 1,8 a 5,7\% dia $^{-1}$ para os experimentos de laboratório, e entre 2,3 e 8,7 \% dia-1 para os experimentos de campo. Gracilaria caudata foi a alga que apresentou as menores taxas, entre $0,8 \mathrm{e}$ 4,9\% dia ${ }^{-1}$, enquanto Hypnea musciformis apresentou taxas de crescimento bastante elevadas, variando entre 5,6 e 15,2\% dia ${ }^{-1}$. Estes resultados são preliminares, e para uma avaliação confiável do potencial produtivo destas algas são necessários experimentos complementares. Entretanto, os valores observados sugerem que estas algas merecem estudos mais detalhados e maior investimento de pesquisa, visando a implementação do cultivo das mesmas na Enseada de Armação do Itapocoroy (Penha, SC).
\end{abstract}

Palavras chave: macroalgas, Rhodophyta, Gracilaria, Hypnea, cultivo, carragenana, ágar, ficocolóides, crescimento

\section{POTENTIAL GROWTH OF CULTIVABLE SEAWEEDS WHICH OCCUR IN ENSEADA DE ARMAÇÃO DO ITAPOCOROY (PENHA, SC): PRELIMINARY EVALUATION}

\begin{abstract}
This work aims to test growth methodology and to evaluate cultivation potential of the red algae Hypnea musciformis (carragenophyte), Gracilaria cervicornis (cf.) and Gracilaria caudata, (agarophytes). In vitro growth was tested for the three algae, and in situ growth was tested for Gracilaria cervicornis (cf.). Preliminary results indicate high growth rates to in situ and in vitro tests, when compared to literature results. Gracilaria cervicornis (cf.) in vitro growth rates ranged from 1,8 to $5,7 \%$ day $^{-1}$, and in situ growth rates ranged from 2,3 to $8,7 \%$ day $^{-1}$. Gracilaria caudata presented lower growth rates, which ranged from 0,8 and $4,9 \%$ day $^{-1}$. Hypnea musciformis presented highest growth rates, which ranged from 5,6 and $15,2 \%$ day $^{-1}$. This results are preliminary. For a good evaluation of productive and cultivation potencial of this algae, complementary tests are needed. However, observed growth rates suggest this algae deserves more research aiming to implement its cultivation on Enseada de Armação do Itapocoroy (Penha, SC).
\end{abstract}

Key Words: macroalgae, Rhodophyta, seaweed, algae, Gracilaria, Hypnea, growth, cultivation, agar, carrageenan, phycocolloid 


\section{INTRODUÇÃO}

A importância das algas marinhas na América Latina como um recurso renovável, vem crescendo significativamente nos últimos anos, especialmente porque seus volumes cada vez maiores de extração geram importantes divisas econômicas para o país produtor (Oliveira, 1990 e 1997). As macroalgas podem ser utilizadas diretamente na alimentação humana, na fabricação de ração animal e de fertilizantes agrícolas, assim como na extração de ficocolóides (ágar, carragenana e alginato) de grande interesse econômico (Oliveira e Plastino, 1992). As principais aplicações dos ficocolóides estão relacionadas com a indústria alimentícia, têxtil, de papel, farmacêutica, entre outras (Jensen, 1993).

A indústria brasileira vem aumentando consideravelmente o consumo de algas e seus derivados, mas a explotação de algas e a produção de ficocolóides no Brasil é relativamente recente e ainda bastante baixa. Entre os anos de 1990 e 1995, o consumo brasileiro de algas e derivados dobrou, mostrando que o mercado vem crescendo cada vez mais (Oliveira, 1997). Entretanto a demanda interna brasileira é suprida principalmente através de importações. A importação de ágar aumentou em $400 \%$ e a importação de alginatos, medicamentos e produtos alimentares derivados de algas, aumentou em $200 \%$, entre 1984 e 1988 (Oliveira e Plastino 1992).

Apesar da baixa produção comercial de algas, há inúmeros bancos naturais e uma ampla diversidade de algas ao longo de toda a costa brasileira. Vários destes bancos são explotados por comunidades locais e vendidos para fabricação nacional de ágar e carragenana ou para exportação (Oliveira e Plastino 1992). Entre as principais algas produtoras de ficocolóides, vários gêneros estão presentes na Enseada de Armação do Itapocoroy (Penha, SC). Dentre eles, dois apresentam elevada abundância: Gracilaria e Hypnea. De acordo com Zertuche-González
(1993), Gracilaria é o gênero responsável por $50 \%$ da produção mundial de ágar. Hypnea está entre os 3 principais gêneros produtores de carragenanas, sendo a única alga utilizada no Brasil para este fim.

Devido à abundância destas algas na Enseada de Armação do Itapocoroy, e ainda devido ao seu potencial econômico, vários projetos estão se iniciando, com o objetivo de desenvolver a tecnologia de cultivo destas algas na região. Um ponto inicial da atividade de cultivo são os estudos sobre as taxas de crescimento das algas e suas respostas às condições ambientais, tanto em termos de crescimento, como em termos de biologia reprodutiva e interações ecológicas.

Este trabalho teve como objetivo testar a metodologia de crescimento e avaliar o potencial de cultivo das algas vermelhas Gracilaria cervicornis (cf.), Gracilaria caudata e Hypnea musciformis, para subsidiar avaliações de crescimento destas e de outras algas que vem sendo estudadas quanto ao potencial produtivo.

\section{METODOLOGIA}

\section{Crescimento in vitro}

Vários exemplares de Gracilaria cervicornis (cf.), Gracilaria caudata e Hypnea musciformis foram coletados na Enseada da Armação do Itapocoroy, Penha, SC (2647' S - $48^{\circ} 37 \mathrm{~W}$ ), em diferentes datas de 1998 . O material coletado foi transportado até o laboratório em caixa térmica com água do ambiente. No laboratório as algas foram cuidadosamente lavadas com água do mar filtrada e mantidas em aquários de $10 \mathrm{~L}$, em água do mar filtrada (filtros de 10,5 e $0,5 \mathrm{~mm}$ ) enriquecida com meio de cultivo comercial Conway (sem adição de silicato), sob aeração constante, em salinidade próxima à do ambiente (» 30 ), temperatura de $20 \circ \mathrm{C}$, fotoperíodo de 16:8 h (claro:escuro), irradiância de » 40 mmol de fótons $\mathrm{m}^{-2} \mathrm{~s}^{-1}$. O meio de cultivo foi trocado semanalmente. 
Para a avaliação do crescimento das algas, ápices dos talos foram seccionados e limpos de epífitas ao microscópio estereoscópico, com o auxílio de pinças e pincéis. Os ápices mais saudáveis e livres de epífitas foram colocados em frascos Erlenmeyer de $500 \mathrm{ml}$, com água do mar filtrada, sob aeração constante, em condições de luz, temperatura e salinidade como descrito anteriormente. Semanalmente as algas foram secas com papel absorvente e pesadas em balança analítica (precisão de 0,001 g) e colocadas em novo meio de cultivo. Os testes de crescimento foram realizados em triplicatas.

O termo crescimento se aplica aos processos fisiológicos e bioquímicos, bem como aos produtos destes processos (Brinkhuis, 1985). O crescimento pode ser expresso como produção de biomassa (rendimento, ou crescimento líquido, que consiste no balanço entre o anabolismo e o catabolismo) ou como taxa de crescimento (Sorokin, 1973). A produção de biomassa é comumente utilizada durante amostragens de rotina, podendo ser expressa como qualquer aumento líquido no peso (seco ou úmido), área, comprimento, volume ou componentes celulares (Brinkhuis, 1985). Os dados de produção de biomassa podem ser transformados em taxa de crescimento, permitindo a avaliação das condições das algas mantidas em cultivo, bem como dos parâmetros que podem ser alterados a fim de melhorar sua performance, sem que os valores absolutos interfiram na interpretação dos resultados. Assim sendo, os resultados dos testes são apresentados em taxas de crescimento relativo.

As taxas de crescimento relativo (TCR) foram calculadas como porcentagem diária de crescimento, utilizando-se as fórmulas de crescimento exponencial (equação 1) e linear (equação 2) sugeridas por Dawes (1998) e descritas abaixo:

$\operatorname{TCR}(\%$ Dia $)=\left(\left(P_{f} / P_{i}\right)^{1 / t}-1\right) \times 100$

(equação 1)
$\operatorname{TCR}(\%$ Dia $\left.)=\left(\left(P_{f}-P_{j}\right) / P_{j}\right) \times 100\right) / t$

(equação 2)

onde: $P_{i}=$ peso inicial, $P_{f}=$ peso final, $t$ = intervalo de tempo entre as medidas, em dias. Os dados de biomassa foram expressos em gramas de peso fresco (g) e o crescimento em \% dia ${ }^{-1}$.

$\mathrm{Na}$ determinação das taxas de crescimento in vitro de Gracilaria cervicornis (cf.) foi realizado um primeiro teste, que avaliou a relação peso/volume (peso de alga por volume de meio de cultivo) mais adequada para os estudos de crescimento. Neste teste foram avaliadas duas condições: uma alta relação peso/volume (média de 13,6 g/L) e uma baixa relação peso/volume (média de 2,6 g/ L). Um segundo teste de crescimento foi realizado para avaliar indícios de limitação por nutrientes durante o intervalo normalmente utilizado para troca de meio (1 troca semanal). Neste experimento, o crescimento de Gracilaria cervicornis (cf.) avaliado em duas situações: com meio de cultivo trocado 1 e 2 vezes por semana.

$\mathrm{Na}$ determinação do crescimento de Gracilaria caudata foram realizados testes com relação peso/volume inicial de $0,16 \mathrm{~g} \mathrm{~L}^{-1}$ e de $0,36 \mathrm{~g} \mathrm{~L}^{-1}$. Na determinação do crescimento de Hypnea musciformis utilizou-se relação peso/volume inicial de aproximadamente $2 \mathrm{~g} \mathrm{~L}^{-1}$. Para ambas as espécies, o meio de cultivo foi trocado semanalmente.

\section{Crescimento in situ}

Para o cultivo de Gracilaria cervicornis (cf.) foi montada uma balsa experimental no parque de cultivo de moluscos marinhos localizado na Enseada da Armação do Itapocoroy, Penha, SC. A estrutura da balsa de cultivo consiste em um quadrado de PVC de $2,25 \mathrm{~m}^{2}$, ao qual foram colocados 6 cabos de polipropileno paralelos (diâmetro de 0,5 $\mathrm{cm}$ ), distanciadas entre si em $20 \mathrm{~cm}$. Em cada cabo foram colocadas mudas de $G$. cervicornis (cf.) de 1 a $3 \mathrm{~g}$, distanciadas entre si em 20 
$\mathrm{cm}$. As mudas foram amarradas ao cabo por um filamento de algodão. Cada muda foi identificada quanto à sua posição na balsa (e.g.: A4 = cabo A, muda 4).

Foram efetuadas 3 coletas: 9, 25 e 43 dias após a montagem da balsa. Para isso, foram selecionadas aleatoriamente 6 mudas, que foram retiradas da balsa, colocadas individualmente em sacos plásticos etiquetados, e conduzidas ao laboratório para pesagem. Os procedimentos de pesagem e os cálculos de crescimento são similares para o crescimento in situ e in vitro.

\section{RESULTADOS PRELIMINARES E DISCUSSÃO}

\section{Crescimento in vitro}

$\mathrm{Na}$ determinação do crescimento de Gracilaria cervicornis (cf.) em diferentes valores de relação peso/volume (peso de alga por volume de meio de cultivo), pode-se observar que a taxa de crescimento diminui com o aumento da relação peso/volume. Quando esta relação foi de »13,6 $\mathrm{g} \mathrm{L}^{-1}$, a taxa de crescimento foi menor, diminuindo com o aumento de biomassa, e variando entre 1,2 e 1,86 $\%$ dia $^{-1}$ (figura 1). Quando esta relação foi de » 2,6 $\mathrm{g} \mathrm{L}^{-1}$, a taxa de crescimento foi superior, variando entre 1,8 e 3,5\% dia-1 (figura 1). Como neste experimento, as taxas de crescimento foram maiores quando a relação peso/ volume foi baixa, nos experimentos subsequentes com Gracilaria optou-se por utilizar baixa biomassa inicial de algas.

Os resultados do crescimento com o meio de cultivo trocado 1 e 2 vezes por semana podem ser observados na figura 2. As taxas de crescimento de Gracilaria cervicornis (cf.) para uma troca semanal de meio de cultivo foram de 2,85 a $5,16 \%$ dia $^{-1}$ (figura 2) e para 2 trocas semanais apresentou uma variação mais ampla, entre 1,82 e 6,4\% dia $^{-1}$. Em ambos os casos, as taxas de crescimento apresentaram uma tendência de decréscimo do início para o final do experimento (figura
2), apesar da baixa relação peso/volume. As taxas de crescimento são bastante similares nos dois experimentos, sugerindo que, para estes valores de biomassa (figura 2A), 0 intervalo de troca de meio pode ser semanal, sem prejuízo para o crescimento das algas. Apesar da relação peso/volume aumentar no decorrer do tempo, a biomassa alcançada parece ser suficientemente baixa para não exaurir os nutrientes durante o intervalo de troca de meio. Possivelmente, para valores de biomassa mais elevados, poderá ser encontrada uma diferença maior nas taxas de crescimento devido ao intervalo de troca de meio. São necessários novos testes variando os valores de biomassa e aumentando o tempo do experimento, para avaliar com segurança esta questão.
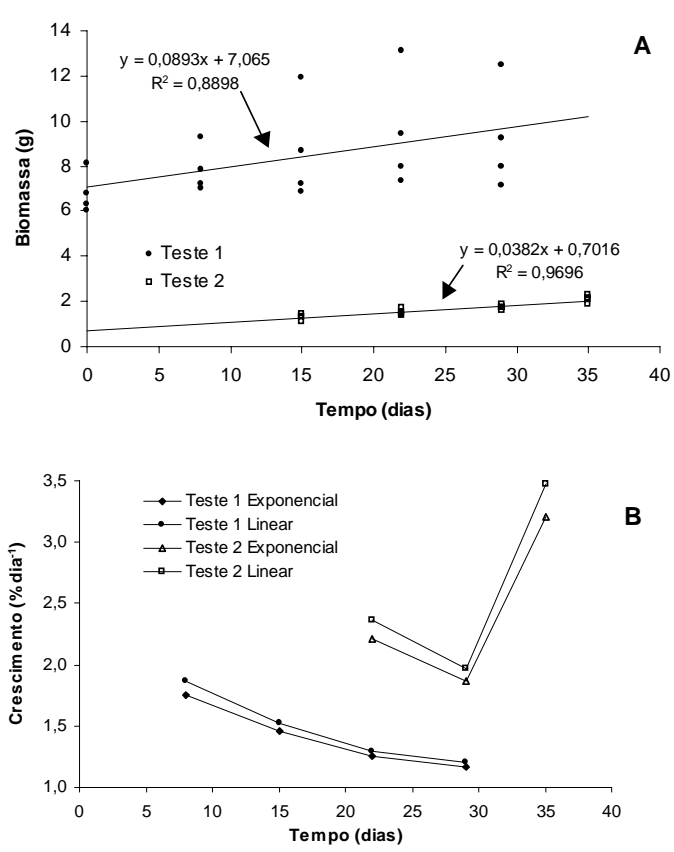

Figura 1: Crescimento de Gracilaria cervicornis (cf.). A: Aumento de biomassa (g) ao longo do tempo, para o teste 1 (relação peso/volume =13,6 g/l) e para o teste 2 (relação peso/volume = 2,6 g/l). B: taxas diárias de crescimento para os testes 1 e 2 , calculadas a partir dos modelos exponencial e linear 
$\mathrm{Na}$ determinação do crescimento de Gracilaria caudata, os dois testes mostraram taxas de crescimento similares, variando entre 0,76 e $4,9 \%$ dia $^{-1}$ (figura 3 ), ambas apresentando uma forte diminuição ao longo do tempo. Na primeira semana do experimento, as taxas de crescimento foram bastante elevadas, e o teste com menor biomassa apresentou crescimento maior (figura 3). A partir da segunda semana, até o final do experimento houve uma inversão desta tendência, e o teste com maior biomassa apresentou maior taxa de crescimento, que inclusive aumentou na última semana de experimento. A queda acentuada nas taxas de crescimento deste experimento pode ser atribuída à contaminação por algas epífitas durante o teste. Um forte
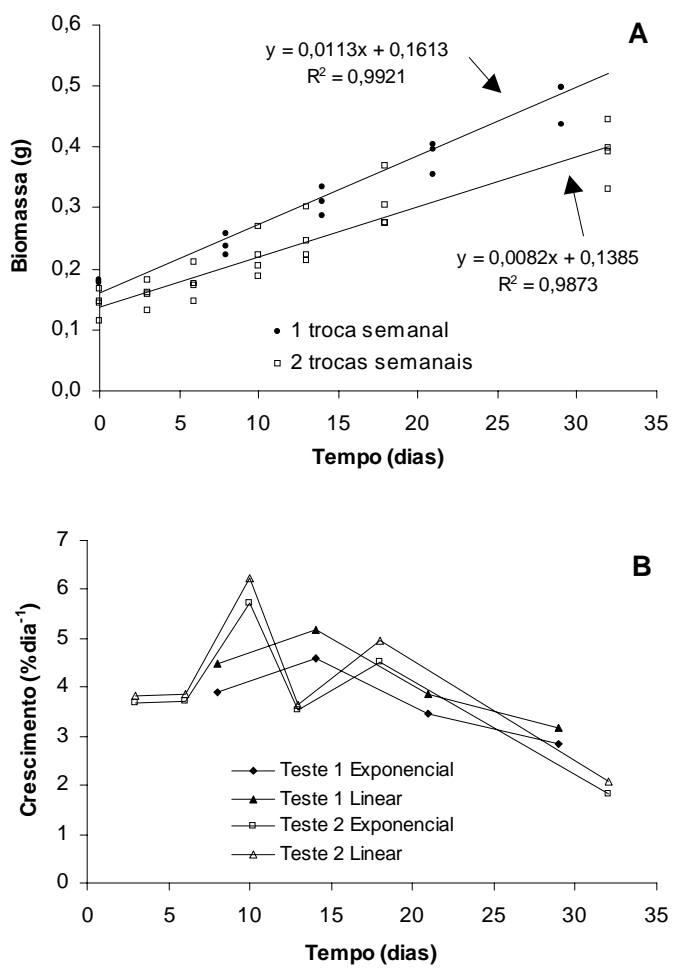

Figura 2: Crescimento de Gracilaria cervicornis (cf.). A: Aumento de biomassa (g) ao longo do tempo, para 1 e 2 trocas semanais do meio de cultivo. B: taxas diárias de crescimento dos dois testes, calculadas a partir dos modelos exponencial e linear epititismo foi observado após 20 dias de cultivo no teste com baixa biomassa e, devido a isso, este teste foi encerrado. No teste com alta biomassa o epifitismo também foi observado, mas de maneira menos intensa. As algas deste experimento foram lavadas com água destilada e ficaram expostas até sofrer uma dessecação superficial, com o objetivo de eliminar as epífitas. Este tratamento funcionou, visto que as epífitas foram eliminadas e a taxa de crescimento aumentou na semana seguinte. Novos experimentos devem ser realizados para avaliar de maneira mais adequada o potencial de crescimento desta espécie.

Em todos os experimentos com Gracilaria cervicornis (cf.) e com Gracilaria
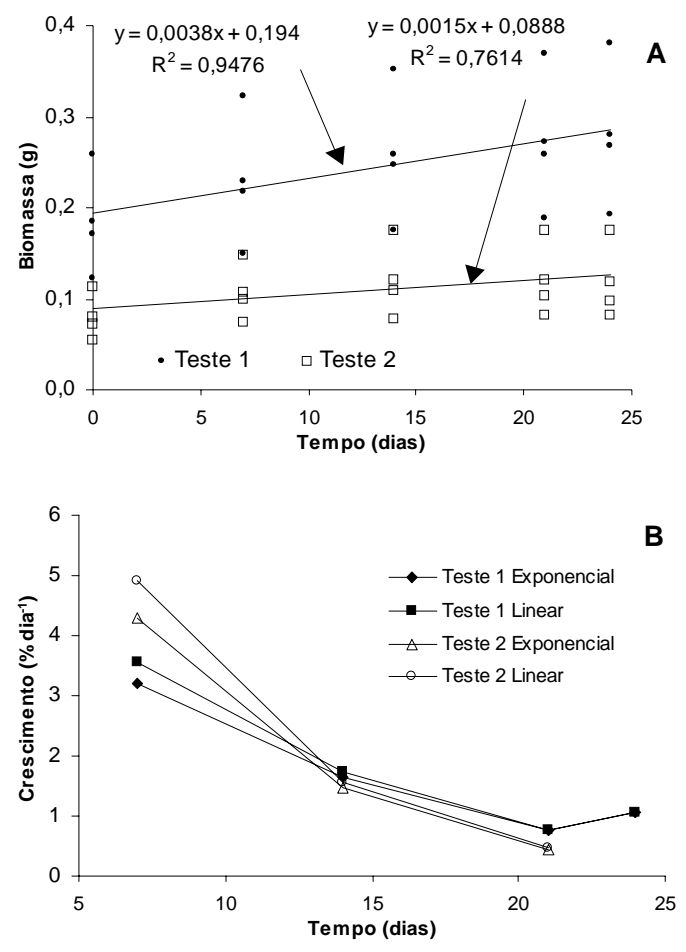

Figura 3: Crescimento de Gracilaria caudata. A: Aumento de biomassa (g) ao longo do tempo, para 2 valores iniciais médios de biomassa: 0,08 e $0,18 \mathrm{~g} / \mathrm{L}$. B: taxas diárias de crescimento os dois testes, calculadas a partir dos modelos exponencial e linear 
caudata a variação da biomassa ao longo do tempo apresentou uma tendência linear de crescimento. Apesar desta tendência, os dados se ajustam tanto ao modelo linear (com maior $r^{2}$ ) como ao modelo exponencial de crescimento (com menor $\mathrm{r}^{2}$ ). $\mathrm{O}$ cálculo do crescimento a partir do modelo linear apresentou valores superiores, mas bastante similares aos apresentados pelo modelo exponencial de crescimento (figuras $1 \mathrm{~B}, 2 \mathrm{~B}$ e $3 \mathrm{~B}$ ), sugerindo que ambos os modelos descrevem adequadamente o crescimento destas algas.

No crescimento de Hypnea musciformis, o aumento de biomassa foi considerável, aumentando em até $800 \%$ no período de 25 dias (figura 4). A biomassa apresentou uma tendência exponencial de crescimento ( $r^{2}$ superior a 99,3\%, figura $4 A$ ). O ajuste da biomassa de Hypnea ao modelo linear de crescimento foi menor $\left(r^{2}=95,8 \%\right)$, sugerindo que, para esta alga, a equação linear não é tão adequada quanto à exponencial para descrever o aumento de biomassa. A taxa diária de crescimento variou entre 5,56 e 15,16\% dia-1 (figura 4B). A taxa de crescimento aumentou no início do experimento, mas diminuiu quando a relação peso/volume superou $6 \mathrm{~g} \mathrm{~L}^{-1}$. Em experimentos posteriores serão testadas diferentes relações peso/volume, assim como intervalo de troca de meio.

\section{Crescimento in situ}

O crescimento de Gracilaria cervicornis (cf.) foi analisado como crescimento real e potencial. $\mathrm{O}$ crescimento real foi calculado utilizando-se todas as algas coletadas no período, inclusive aquelas que apresentaram crescimento nulo ou negativo, devido às perdas de biomassa por quebra da fronde. $O$ crescimento potencial foi calculado excluindo-se as algas que sofreram perda de biomassa no período. Os resultados deste experimento podem ser observados na tabela 1. Como a quebra de algas é uma situação relativamente comum, relacionada principal- mente às condições de hidrodinâmica do local, para avaliar o rendimento líquido do cultivo, deve ser considerado o crescimento real. Entretanto, se as algas que sofreram perda de biomassa são excluídas do cálculo, podemos avaliar o potencial produtivo da espécie.

O crescimento diário real apresentou taxas variando entre 2,03 e 5,66 \% dia $^{-1}$, que são valores bastante similares aos observados para Gracilaria cervicornis (cf.) nos experimentos de laboratório (figuras 1 e 2, tabela 2). O crescimento diário potencial apresentou valores elevados (tabela 1) sugerindo que a otimização da metodologia pode melhorar o rendimento obtido neste cultivo experimental. O ganho em biomassa foi considerável, e a alga obteve, em 25 dias de crescimento, um aumento biomassa de $216 \%$ com relação à biomassa inicial. Em 42 dias de crescimento, houve um aumento de $292 \%$. Entretanto, neste último período o epifitismo foi
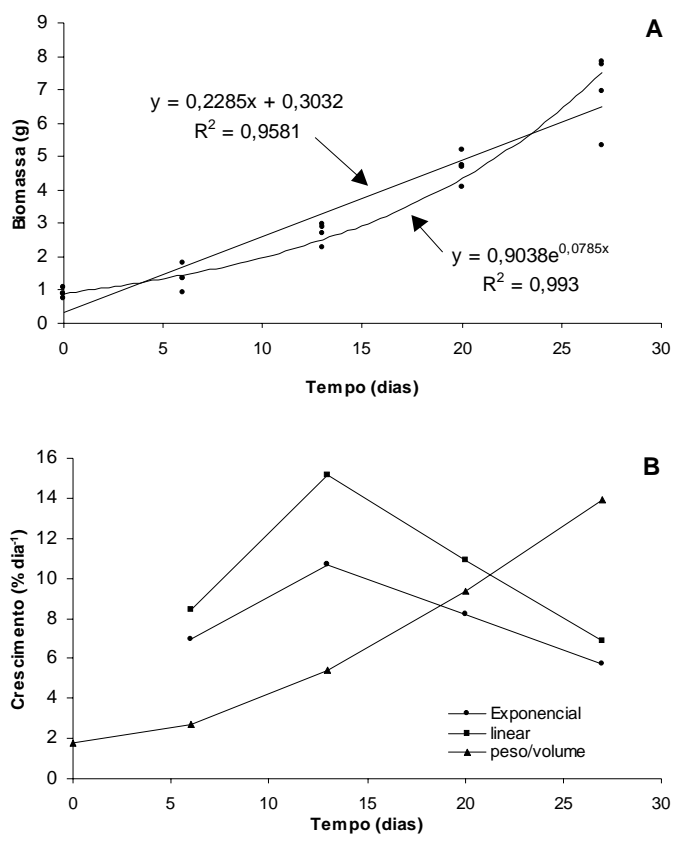

Figura 4: Crescimento de Hypnea musciformis. A: Aumento de biomassa (g) ao longo do tempo. B: taxas diárias de crescimento calculadas a partir dos modelos exponencial e linear, e a relação peso/volume no experimento 
bastante elevado, o que reduziu a taxa diária de crescimento (tabela 1). A presença de epífitas e epifauna torna-se um problema, pois diminui a disponibilidade de luz e nutrientes e dificulta as trocas gasosas, o que reduz o crescimento da planta. Outro problema de ordem prática é o esforço e o gasto de tempo para limpar as algas dos organismos epifíticos. Com estes resultados preliminares, é possível supor que o intervalo de tempo mais adequado para a coleta das algas seria em torno de 25 dias. Entretanto, experimen-

Tabela 1: Crescimento de Gracilaria cervicornis (cf.) em balsa de cultivo, na Enseada de Armação do Itapocoroy: percentual de algas quebradas em cada coleta, taxa diária de crescimento (\% dia-1 $)$, ganho percentual de biomassa com relação à biomassa inicial, durante o intervalo amostral.

\begin{tabular}{|c|c|c|c|}
\hline Intervalo de crescimento (dias) & 9 & 25 & 43 \\
\hline Algas quebradas (perdas) (\%) & 42,8 & 33,3 & 16,7 \\
\hline $\begin{array}{l}\text { Crescimento diário médio real em } \% \text { dia }^{-1} \text { (incluindo as } \\
\text { perdas) }\end{array}$ & $2,03 \pm 4,4$ & $5,66 \pm 5,1$ & $5,54 \pm 4,7$ \\
\hline $\begin{array}{l}\text { Crescimento diário médio potencial em } \% \mathrm{dia}^{-1} \\
\text { (excluindo as perdas) }\end{array}$ & $5,1 \pm 2,2$ & $8,67 \pm 2,6$ & $6,96 \pm 3,5$ \\
\hline $\begin{array}{l}\text { Ganho percentual real com relação à biomassa inicial } \\
\text { (incluindo as perdas) }\end{array}$ & 18,28 & 141,57 & 232,7 \\
\hline $\begin{array}{l}\text { Ganho percentual potencial com relação à biomassa } \\
\text { inicial (excluindo as perdas) }\end{array}$ & 45,92 & 216,85 & 292,4 \\
\hline
\end{tabular}

Tabela 2: Ganho percentual de biomassa com relação à biomassa inicial, para cada intervalo amostral.

\begin{tabular}{ccccc}
\hline $\begin{array}{c}\text { Intervalo amostral } \\
\text { (dias) }\end{array}$ & $\begin{array}{c}\text { G. cervicornis (cf.) } \\
\text { situ) }\end{array}$ & $\begin{array}{c}\text { G. cervicornis } \\
\text { (in vitro) }\end{array}$ & $\begin{array}{c}\text { G. caudata (in } \\
\text { vitro) }\end{array}$ & $\begin{array}{c}\text { H. musciformis (in } \\
\text { vitro) }\end{array}$ \\
\hline 6 & & 24,5 & 30,5 \\
7 & & 35,9 & \\
8 & $45,9 \%$ & & & \\
9 & & 55,7 & \\
10 & & 73,1 & \\
13 & & 78,2 & \\
14 & 116,2 & 48,8 & \\
18 & & 126,9 & \\
21 & & 53,8 & \\
24 & & 59,9 & \\
25 & & & \\
27 & & & \\
29 & & & \\
34 & & & \\
43 & & 185,0 & \\
\hline
\end{tabular}


Tabela 3: Taxa diária de crescimento e rendimento em ficocolóides para diferentes espécies de algas e diferentes técnicas e cultivo ou coleta.

\begin{tabular}{|c|c|c|c|c|}
\hline Espécie & Tipo de cultivo & $\begin{array}{c}\text { Cresc, \% } \\
\text { dia }^{-1}\end{array}$ & $\begin{array}{c}\text { Rendim, } \\
\text { colóides \% }\end{array}$ & Autores \\
\hline Gracilaria cervicornis (cf) & Campo & 2,3 a 8,7 & & Presente trabalho \\
\hline Gracilaria cervicornis (cf) & Laboratório & 1,8 a 5,7 & & Presente trabalho \\
\hline Gracilaria caudata & Laboratório & 0,8 a 4,9 & & Presente trabalho \\
\hline Hypnea musciformis & Laboratório & 5,6 a 15,2 & & Presente trabalho \\
\hline Gracilaria edulis & Campo & 1,1 a 1,9 & & Kaladharam et al,, 1996 \\
\hline Gracilaria cervicornis & coleta & & 27 a 31 & Aponte-Dias \& Lemus-Castro, 1988 \\
\hline Gracilaria cornea & Campo & 1,7 a 2,4 & 24 & Rincones Leon, 1988 \\
\hline Gracilaria chilensis & tanques & 0,4 a 1,3 & & Oliveira et al.,1990 \\
\hline Gracilaria sp, & tanques & 0,7 a 0,9 & 7,8 a 14,7 & Macchiavello, 1990 \\
\hline Gracilariopsis bailinae & tanques & 0,2 a 1,1 & 6 a 14 & Hurtado-Ponce \& Pondevida, 1997 \\
\hline Gracilaria bursa-pastoris & Campo & 0,3 a 4 & & Casabianca et al., 1997 \\
\hline
\end{tabular}

tos mais completos devem ser desenvolvidos para a definição de uma metodologia adequada de cultivo e manejo destas algas.

\section{O potencial de cultivo das algas}

Os experimentos já desenvolvidos tem sua maior contribuição no treinamento de metodologias do que nos resultados propriamente ditos, os quais são preliminares. Apesar disso, estes resultados são animadores quanto à perspectiva de cultivo de algas produtoras de ficocolódes na Enseada de Armação do Itapocory. Hypnea musciformis, produtora de carragenana, é a alga que apresenta as maiores taxas de crescimento, chegando a aumentar em torno de $700 \%$ com relação à biomassa inicial, em 27 dias de cultivo (tabela 2). Gracilaria cervicornis (cf.), produtora de ágar, apresenta, ao final de 43 dias, um aumento de $280 \%$ (em laboratório) a 290 $\%$ (em campo) com relação à biomassa inicial (tabela 2). Comparando-se as taxas diárias de crescimento obtidas no presente trabaIho, com taxas de crescimento da literatura (tabela 3), as perspectivas de cultivo tornamse ainda mais atrativas. Entre as várias espécies de Gracilaria observadas na literatu$\mathrm{ra}$, as taxas de crescimento variam entre 0,2 e $4 \%$ dia $^{-1}$, sendo que este último valor ( $4 \%$ $\mathrm{dia}^{-1}$ ) só foi encontrado em um trabalho com
Gracilaria bursa-pastoris (Casabianca et al., 1997).

Para uma avaliação confiável do potencial produtivo das algas, estão em andamento vários testes, com o objetivo de avaliar a resposta das algas a diferentes temperaturas, salinidades e intensidades luminosas. Entre estes, tem grande importância os testes realizados em campo, para avaliar a metodologia adequada para o cultivo, em termos de estrutura física, de densidade e profundidade adequadas, alem da periodicidade ideal de coleta, entre outros fatores.

\section{AGRADECIMENTOS}

Agradecemos aos professores Charrid Resgalla e Leonardo Rorig, pela orientação técnica e por ceder espaço e equipamento para a execução dos trabalhos de laboratório. Agradecemos também aos técnicos do CTTMar/Univali, pelo apoio durante os trabaIhos de campo.

\section{REFERÊNCIAS BIBLIOGRÁFICAS}

Aponte Diaz, M. \& Lemus Castro, A.L. Comparative study of the agar obtained from three Gracilaria species feasible for culture in Venezuela, p. 117-119. In: 
E.C.de; Kautsky, N. (eds.), Cultivation Of Seaweeds In Latin America.Oliveira

Brinkhuis, B.H. 1985. Growth patterns and rates, p. 461-477. In: Littler, M.M. e Littler, D.S. (eds), Handbook of phycological methods - ecological field methods: macroalgae. Cambridge University Press. Casabianca, M.L.D., Marinho-Soriano, E., \& Laugier, T. 1997. Growth of Gracilaria bursa-pastoris in a Mediterranean Lagoon: Thau, France. Bot.Mar. 40:29-37. Dawes, C.J. 1998. Marine Botany. 2nd.Ed. John Wiley \& Sons, New York, 480pp.

Hurtado-Ponce, A.Q. \& Pondevida, H.B. 1997. The interactive effect of some environmental factors on the growth,agar yield and quality of Gracilariopsis bailinae (Zhang et Xia) cultured in tanks. Bot.Mar. 40:217-223.

Jensen, A. 1993. Present and future needs for algae and algal products. Hidrobiologia, 260/261:15- 23.

Kaladharan, P., Vijayakumaran, K., \& Chennubhotla, V.S.K. 1996. Optimization of certain physical parameters for the mariculture of Gracilaria edulis (Gmelin) silva in Minicoy lagoon (Laccadive Archipelago). Aquaculture 139:265-270.

Macchiavello, J.E. 1990. Culture of Gracilaria, p. 59-63. In: E.C.de; Kautsky, N. (eds.), Cultivation Of Seaweeds In Latin America.Oliveira.

Oliveira, E.C. 1990. The rationale for seaweed cultivation in South America, p. 135-141. In: E.C.de; Kautsky, N. (eds.), Cultivation Of Seaweeds In Latin America.Oliveira
Oliveira, E.C. 1997. Algas marinhas: um recurso ainda pouco explotado pelo Brasil. Panorama da Aquicultura 6/7:24-26.

Oliveira, E.C. \& Plastino, E. M. 1992. The exploitation of seaweeds in Braxil - The need for a new code to assure sustainabel yelds, p. 83-98. In: Cordeiro-Marino, M.; Azevedo M.T. P.; Sant'anna, C.L.; Tomita, N. Y. (Eds.). Algae and environment: a general approach. Sociedade Brasileira de Ficologia.

Oliveira, E.C.D., Paula, E.J.D., \& Berchez, F.A.S. 1990. Essays on the cultivation of tropical red seaweeds in tanks, p. 79-87. In: E.C.de; Kautsky, N. (eds.), Cultivation Of Seaweeds In Latin America.Oliveira.

Rincones Leon, R.E. 1990. Experimental cultivation of an agarophyte algae: Gracilaria cornea in the northwest coast of Venezuela, p. 65-67. In: E.C.de; Kautsky, N. (eds.), Cultivation Of Seaweeds In Latin America.Oliveira.

Sorokin, C. 1973. Dry weight, packed cell volume and optical density, p. 321-343. In: Stein, J.R. (ed) Handbook of phycological methods - culture methods and growth measurements. Cambridge University Press.

Zertuche - Gonzáles, J. A. 1993. Situación actual del cultivo de algas agaraofitas en America Latina y el Caribe. FAO-ITALIA. Proyecto Aquila II. Programa Cooperativo Gubernamental. Documento de campo n. 13: p. 5 - 15. 